\title{
Study on critical liquid-carrying flow model in inclined Wells
}

\author{
Quanhua Huang ${ }^{1}$, Xingyu Lin ${ }^{1 *}$, Yunjun $\mathrm{He}^{2}$, Chengyin Wang ${ }^{3}$, Yi Hu ${ }^{4}$, Hongjun Ding ${ }^{1}$, Jian Jiang ${ }^{1}$, Jian $\mathrm{Li}^{1}$ \\ ${ }^{1}$ Petroleum engineering school, Southwest petroleum university, Chengdu, Sichuan, 610500, china \\ ${ }^{2}$ Natural gas laboratory, Daqing oilfield exploration and development research institute, Daqing, Heilongjiang, 16300, china \\ ${ }^{3}$ East China Petroleum Bureau, Sinopec, Nanjing, Jiangshu, 210019, China \\ ${ }^{4}$ Central China Branch,China Petrochemical Sales Co.LTD.Wuhan,Hubei,430021, China
}

\begin{abstract}
It is very important to calculate the critical liquid-carrying flow for gas well.In this paper, an ellipsoid droplet model is established. By analyzing the droplet force in the inclined well and comprehensively considering the droplet drag force, gravity force, friction force, buoyancy force and supporting force, the general formula for calculating the critical liquid carrying flow is obtained. By using the reasonable formula of gas-liquid interfacial tension and The Formula of Weber number, the critical liquid carrying flow of gas well is verified.
\end{abstract}

\section{Introduction}

In the calculation of the critical liquid-carrying flow of the gas well, it is necessary to consider the most difficult way for the droplet to be removed from the gas well, which includes the maximum droplet diameter and the friction between the droplet and the wellbore. In 1969, TUNER ${ }^{[1]}$ developed a circular droplet model suitable for vertical Wells to calculate the critical liquid-carrying flow. Nosseir and Coleman et al ${ }^{[2-3]}$ made corrections on this basis to improve the calculation accuracy. In China, there are many relevant models for calculating the liquidcarrying flow in vertical Wells, and relevant improvements have been made, such as ellipsoid, spherical cap-shaped. For example, Li Min model, Wang Yizhong model and Dai Yunlong model was established ${ }^{[4-}$ ${ }^{6}$.For the study of critical liquid-carrying flow in inclined Wells, Ming Ruiqing proposed a spherical model considering friction force and Xiong Yu proposed an ellipsoid droplet critical liquid-carrying flow calculation model. On this basis, a new calculation model is established by considering the influence of various parameters on the critical liquid carrying flow.

Considering the droplet deformation in a vertical well, an ellipsoidal droplet model was established by Xiong $\mathrm{Yu}^{[7]}$ :

$$
\begin{gathered}
v_{c}=C_{k, W_{e c}}\left(\frac{\sigma\left(\rho_{l}-\rho_{g}\right)}{\rho_{g}^{2}}\right)^{0.25} \\
C_{k, W_{e c}}=\left(\frac{4 g W_{e c}}{3 k^{2} C_{D}}\right)^{0.25}
\end{gathered}
$$

A calculation method of critical liquid-carrying velocity in inclined Wells based on ellipsoidal droplet model was established by Du Shejiao ${ }^{[8]}$ :

$$
\begin{aligned}
& v_{c}=C_{k, W_{e c}}\left(\frac{\sigma\left(\rho_{l}-\rho_{g}\right)}{\rho_{g}^{2}}\right)^{0.25} \\
& C_{k, W_{e c}}=\left(\frac{4 g W_{e c}}{3 k^{2} C_{D}} \sin \theta\right)^{0.25}
\end{aligned}
$$

Ming Ruiqing ${ }^{[9]}$ believed that the collision between droplet and wellbore could not be ignored in the calculation of the critical liquid-carrying flow flow, and a spherical calculation model was established:

$$
v_{c}=1.9\left(\frac{W_{e c} \sigma\left(\rho_{l}-\rho_{g}\right) \sin \theta+\lambda\left(\rho_{l}-\rho_{g}\right) \cos \theta}{C_{d} \rho_{g}^{2}}\right)^{0.25}
$$

\section{Establishment and solution of the model}

On the basis of existing theories, an ellipsoidal droplet model considering the droplet collision with the wellbore was established, with the following assumptions:

1. The extrusion and collision between droplets are ignored.

2. The droplet is ellipsoid, the section parallel to the pipe string is ellipsoid, and the section perpendicular to the pipe holding direction (windward side) is circular.

3. Droplet deformation is symmetric along the central axis

*Corresponding authors e-mail: 364520856@@q.com 


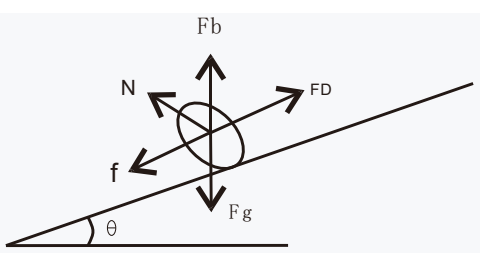

Fig1 Droplet model

Critical liquid-carrying flow can be understood as the flow required for the gas to fully bring the liquid from the bottom to the surface, usually referring to the largest droplet, while the collision of the droplet with the wellbore should also be considered. The droplet on the wellbore is under the combined action of gravity, buoyancy, drag force, supporting force and friction force, and reaches equilibrium under the critical condition (Fig1).

$$
\begin{gathered}
N+F_{\mathrm{b}} \cos \theta=F_{\mathrm{g}} \cos \theta \\
F_{\mathrm{D}}+F_{\mathrm{b}} \sin \theta=F_{\mathrm{g}} \sin \theta+f
\end{gathered}
$$

Gravity, buoyancy, drag force and friction force can be expressed as:

$$
\begin{gathered}
F_{\mathrm{g}}=\rho_{1} \mathrm{~g} V=\rho_{1} \mathrm{~g}\left(\frac{\pi}{6} d_{0}^{3}\right) \\
F_{b}=\rho_{\mathrm{g}} \mathrm{g} V=\rho_{\mathrm{g}} \mathrm{g}\left(\frac{\pi}{6} d_{0}^{3}\right) \\
F_{\mathrm{D}}=\frac{1}{2} C_{\mathrm{D}} S \rho_{\mathrm{g}} v_{\mathrm{c}}^{2} \\
f=\lambda N=\lambda\left(F_{\mathrm{g}} \cos \theta-F_{\mathrm{b}} \cos \theta\right)
\end{gathered}
$$

Combine the above expressions and solve:

$$
v_{c}=\left(\frac{2\left(\rho_{l}-\rho_{g}\right) g V(\lambda \cos \theta+\sin \theta)}{C_{D} S \rho_{g}}\right)^{0.25}
$$

Ratio of the volume of the ellipsoid droplet after deformation to the cross section of the windward side:

$$
\frac{V}{S}=\frac{\frac{\pi}{6} d_{0}^{3}}{\frac{\pi}{4} d_{1}^{2}}=\frac{2 d_{0}}{3 k^{2}}
$$

$k$ Is the characteristic parameter of droplet maximum deformation, $d$ Is the maximum diameter of the windward side,$d_{0}$ Is the initial diameter of the droplet:

$$
k=\frac{d_{1}}{d_{0}}
$$

Maximum droplet diameter before droplet deformation:

$$
d_{\max }=\frac{W_{e c} \sigma}{\rho_{g} v_{c}^{2}}
$$

Therefore, there are:

$$
v_{c}=C_{k, W_{e c}}\left(\frac{\sigma\left(\rho_{l}-\rho_{g}\right)}{\rho_{g}^{2}}\right)^{0.25}
$$

Where,

$$
C_{k, W_{e c}}=\left(\frac{4 g W_{e c}}{3 k^{2} C_{D}}(\lambda \cos \theta+\sin \theta)\right)^{0.25}
$$

The empirical formula is used to calculate the surface tension of gas-liquid ${ }^{[10-11]}$ :

$$
\begin{gathered}
\sigma_{g w}(p, T)=\frac{1.8(410.93-T)}{206}\left(\sigma_{1}-\sigma_{2}\right)+\sigma_{2} \\
\sigma_{1}=76 e^{-0.036257 p} \\
\sigma_{2}=52.5-0.87018 p
\end{gathered}
$$

$\sigma$ _— surface tension, $\mathrm{mN} / \mathrm{m} ; \sigma_{1}$ — Water surface tension at $23.33^{\circ} \mathrm{C}, \mathrm{mN} / \mathrm{m} ; \sigma_{2}$ — Water surface tension at $137.78^{\circ} \mathrm{C}, \mathrm{mN} / \mathrm{m} ; T$ - Temperature, $\mathrm{K} ; p$ Pressure, $\mathrm{MPa}$ 。

Oil-gas surface tension;

$\sigma_{\text {og }}=[42.4-0.047(1.8 \mathrm{~T}-459.67)-0.267 \mathrm{API}] \exp (-0.10152 \mathrm{p})$

Gas-liquid surface tension:

$$
\sigma=\sigma_{\mathrm{og}} \frac{Q_{o}}{Q_{o}+Q_{w}}+\sigma_{g w} \frac{Q_{w}}{Q_{o}+Q_{w}}
$$

Wang Zhibin ${ }^{[12]}$ proposed the general relation of Weber number:

$W_{e c}=25\left(k^{2}+\frac{1}{k^{4}}-2\right) /\left(7.951-\frac{2.744}{k^{2}}+\frac{0.3077}{k}-5.117 k+0.501 k^{2}\right)$

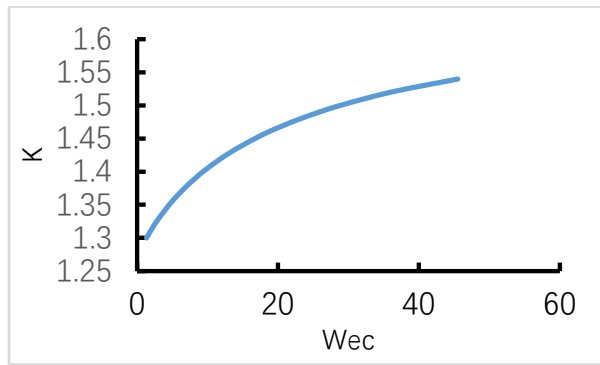

Fig. 2 Relation between deformation coefficient and critical Weber number

Ellipsoid height parallel to the wellbore:

$$
h=\frac{d_{0}}{k^{2}}
$$

Using wang Zhibin's ellipsoid surface area calculation formula: 


$$
A_{1}=2 \pi\left[\left(\frac{d}{2}\right)^{2}+\left(\frac{h}{2}\right)^{2}\right]=\frac{1}{2} \pi\left(k^{2}+\frac{1}{k^{4}}\right) d_{0}^{2}
$$

Reza Barati ${ }^{[13]}$ used the polygene genetic code (GP) method to introduce the optimization results of hyperbolic tangent function and calculate the drag coefficient $C_{D}$ :

$$
\begin{aligned}
& C_{D}=5.4856 \times 10^{9} \tanh \left(\frac{4.3374 \times 10^{-9}}{\operatorname{Re}}\right)+0.0709 \tanh \left(\frac{700.6574}{\operatorname{Re}}\right) \\
& +0.3894 \tanh \left(\frac{74.1539}{\operatorname{Re}}\right)-0.1198 \tanh \left(\frac{7429.0834}{\operatorname{Re}}\right) \\
& +1.7174 \tanh \left(\frac{9.9851}{\operatorname{Re}+2.3348}\right)+0.4744
\end{aligned}
$$

General formula for the calculation of critical liquid carrying flow:

$$
q_{c}=2.5 \times 10^{8} \frac{A P v_{c}}{Z T}
$$

Where, A-Gas well string cross-sectional area, $\mathrm{m}^{2}$ 。

\section{Model analysis:}

The relationship between deformation coefficient $\mathrm{K}$ and parameters $C_{k, W_{e c}}$ in this model:

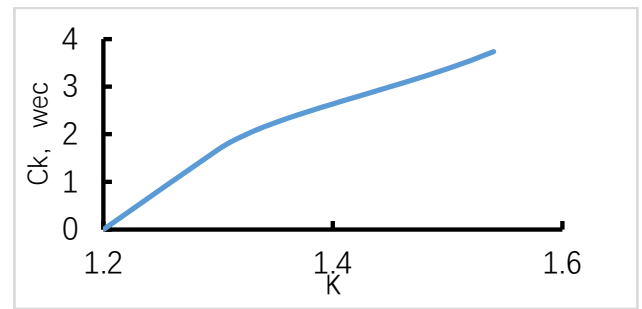

Fig. 3 Relation diagram of deformation coefficient and

$$
\text { parameters } C_{k, W_{e c}}
$$

Under different deformation coefficients, there are different critical Weber coefficients. Compared with Xiong's model, the corresponding critical liquid carrying flow in this paper is relatively small, which is due to the consideration of well inclination and friction between wellbore. The friction coefficient is 0.1 , and the relationship is as follows:

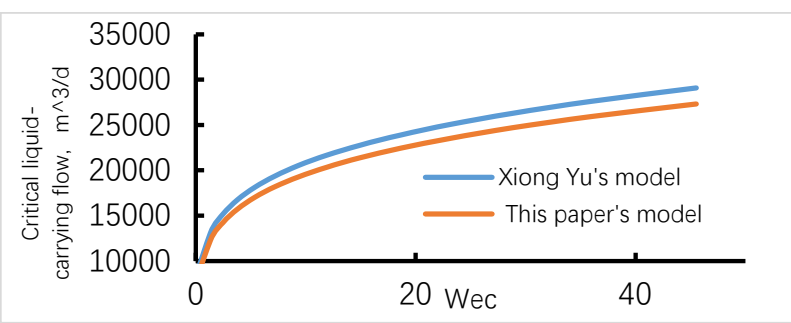

Fig. 4 The critical carrying liquid flow at different Weber coefficients
Under different Angle, compared with Ming's model, in this paper, the corresponding critical carrying-liquid flow is relatively small too, this is because the Ming's model did not consider droplet deformation in the wellbore. In this paper, we consider the droplet model for ellipsoid, which is closer to the real underground environment. In the case, compared with Ming's model (fig 5) when the deformation coefficient $\mathrm{k}$ is 1.3.It can be seen from fig 5 that the larger the Angle is, the Well type more tend to the vertical, the higher the critical carryingliquid flow is. This is because the larger the angle is, the smaller the inclination of the wellbore is, the smaller the corresponding friction force is, and the greater the critical liquid carrying capacity is.

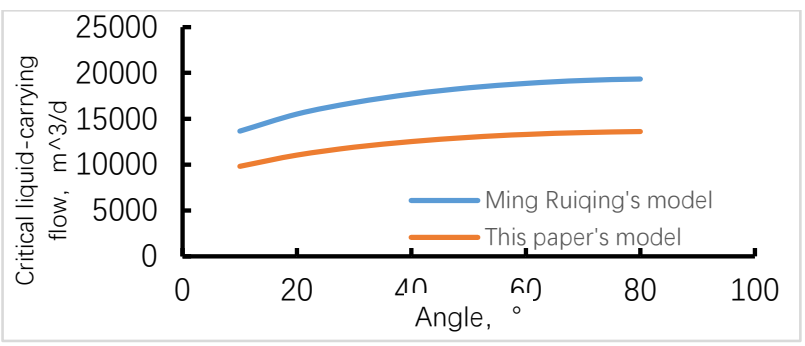

Fig. 5 The critical carrying liquid flow at different angles

\section{Conclusion:}

According to the liquid distribution characteristics and movement characteristics in liquid-gas Wells, an ellipsoid droplet model is established, and a critical liquid-carrying flow calculation model for condensate Wells is obtained by considering the droplet contact with the inclined wellbore. Compared with other models, the ellipsoid model and the friction force between the wellbore and the droplet are more scientific. The results show that the model can be well applied to the actual production process of inclined well to predict the critical carrying-liquid flow.

\section{Reference}

1. TURNER, R G, HUBBARD, M G, DUKLER, A E. (1969) Analysis and prediction of minimum flow rate for the continuous removal of liquids from gas wells. Journal of Petroleum Technology, 21(11): 1475-1482.

2. Coleman, S B. (1991) A New Look at Predicting Gas Well Load Up. JPT, 329-333.

3. Nosseir, M A. (1997) A New Approach for Accurate Prediction of Loading in Gas Well Under Different FlowingConditions. SPE 37408.

4. Li ,M, Sun, L, Li ,S L,et al. (2001) New view on continuous removal Liquids from Gas Wells. SPE 70016.

5. Dai, Y L, Hu, Y Y, Zhang, C Y. (2016) Study on the law of fluid transport in gas Wells. Liaoning chemical industry, 45(06):779-781.

6. Wang, Y Z, Liu, Q W (2007). A new method for calculating the minimum fluid critical flow in gas Wells. Daqing Petroleum Geology and Development, 
26 (6): 82-85.

7. Xiong, Y,Zhang, M M, Lu, Y.(2015) A forecasting gas well both liquid continuous critical condition of universal model. Water dynamics research and progress in A series, 30 (02) : 215-222.

8. Du, S J, Liang, Y, Wang, W J, Qiu, D J. (2019) A new model for predicting the critical fluid flow in inclined gas Wells. Journal of petroleum and natural gas chemistry, 48(03):72-77.

9. Ming, R Q, et al. (2019)A general model for predicting the critical fluid carrying rate in condensate Wells.Drilling and Production Technology, 42(6) : 69-72

10. Yang, J S. Basis of Gas recovery process. (1994) Beijing: Petroleum Industry Press, 330-334.

11. Chen, Y Q. (1990)Practical method of Oil and Gas reservoir Engineering. Beijing: Petroleum Industry Press, 442-452.

12. Wang, Z B, LI, Y C. (2012) Mechanism of continuous liquid transport in gas Wells. Acta Petroleum Sinica, 33(4): 681-686.

13. BARATI, R. Development of empirical models with high accuracy for estimation of drag coefficient of flow around a smooth sphere: An evolutionary approach. Powder Technology, 2014, (257): 11-19. 